\title{
Strain Effects on Phase Transitions in Transition Metal Dichalcogenides
}

\author{
Seoung-Hun Kang, Young-Kyun Kwon* \\ Department of Physics and Research Institute for Basic Sciences, Kyung Hee University, Seoul, \\ 02447, Korea
}

Korea Institute for Advanced Study (KIAS), Seoul 02455, Korea

\begin{abstract}
We perform density functional theory calculation to investigate the structural and electronic properties of various two-dimensional transition metal dichalcogenides, $\mathrm{MX}_{2}(\mathrm{M}=\mathrm{Ti}, \mathrm{V}, \mathrm{Cr}, \mathrm{Zr}, \mathrm{Nb}, \mathrm{Mo}, \mathrm{Hf}$, Ta, or $\mathrm{W}$, and $\mathrm{X}=\mathrm{S}$ or Se), and their straininduced phase transitions. We evaluate the relative stability and the activation barrier between the octahedral- $T$ and the trigonal- $H$ phases of each $\mathrm{MX}_{2}$. It is found that the equilibrium and phase transition characteristics of $\mathrm{MX}_{2}$ can be classified by the group to which its metal element $\mathrm{M}$ belongs in the periodic table. $\mathrm{MX}_{2}$ with $\mathrm{M}$ in the group 4 ( $\mathrm{Ti}, \mathrm{Zr}$, or $\mathrm{Hf})$, forms an octahedral- $T$ phase, while that with an $\mathrm{M}$ in the group $6(\mathrm{Cr}, \mathrm{Mo}$, or $\mathrm{W})$ does a trigonal- $H$ phase. On the other hand, $\mathrm{MX}_{2}$ with $\mathrm{M}$ in the group $5(\mathrm{~V}, \mathrm{Nb}, \mathrm{Ta})$, which is in-between the groups 4 and 6 , may form either phase with a similar stability. It is also found that their electronic structures are strongly correlated to the structural configurations: mostly metallic in the $T$ phase, while semiconducting in the $H$ phase, although there are some exceptions. We also explore the effects of an applied stress and find for some $\mathrm{MX}_{2}$ materials that the resultant strain, either tensile or compressive, may induce a structural phase transition by reducing the transition energy barrier, which is, in some cases, accompanied by its metal-insulator transition.
\end{abstract}

Keywords: Transition metal dichalcogenides; Phase transition; Strain effect; Density functional theory

\footnotetext{
${ }^{*}$ Corresponding author.

Email address: ykkwon@khu . ac .kr (Young-Kyun Kwon)
} 


\section{Introduction}

Transition metal dichalcogenides (TMDCs) in the form of a monolayer have emerged as they have been easily exfoliated to a single layer due to the weak interlayer interaction like in graphene. One of the unique properties of TMDCs is polymorphism with distinct electronic properties. The relative arrangement of chalcogen atoms to the transition metal atom determines the structural symmetry, and thus the phase, trigonal prismatic $H\left(\mathrm{D}_{3 h}\right)$ or octahedral $T$ phase $\left(\mathrm{O}_{h}\right)$. Although there are a variety of combinations in TMDCs, most TMDC studies have focused mainly on $\mathrm{MoS}_{2}$. It was interestingly revealed that $\mathrm{MoS}_{2}$ can have completely different electronic characteristics that are semiconducting and metallic in the $H$ and $T$ phases, respectively. [1, 2, 3] It was also observed in $\mathrm{MoS}_{2}$ that both $H$ and $T$ phases could coexist in a certain condition, [4] and metal-insulator transitions were induced by strain. [5, 6] Furthermore, it was reported that $\mathrm{MoS}_{2}$ has many useful properties such as electronic, optical, mechanical, and chemical properties as well as thermal properties, [7, 8, 9] which were demonstrated by $\mathrm{MoS}_{2}$-based electrical and photoelectronic devices. [10, 11] Some other TMDC materials, such as $\mathrm{MoSe}_{2}, \mathrm{WS}_{2}, \mathrm{WSe}_{2}$, were also used to study their various physical properties. [11, 12]

Recent experimental and theoretical studies have endeavored to control the electronic property of $\mathrm{MoS}_{2}$ through an electron doping from intercalated alkali atoms, [2, 13, 14, 15, 16], in-plane strain [6, 16] or the substitutional doping of Re atoms. [17] Although it has been reported that the $\mathrm{MoS}_{2}$ band gap can be changed by strain [18] or hydrostatic pressure, [19] there have been no systematic studies on the electronic characteristics of various single-layered TMDCs affected by structural deformations such as strain.

In this paper, we report a first-principles study of the structural and electronic properties of $\mathrm{MX}_{2}(\mathrm{M}=\mathrm{Ti}, \mathrm{V}, \mathrm{Cr}, \mathrm{Zr}, \mathrm{Nb}, \mathrm{Mo}, \mathrm{Hf}, \mathrm{Ta}$, or $\mathrm{W}$, and $\mathrm{X}=\mathrm{S}$ or $\mathrm{Se})$ and their phase transitions. Our study revealed that each $\mathrm{MX}_{2}$ has a preferred phase or either phase depending on the group to which its metal atom $\mathrm{M}$ belongs in the periodic table. For the phase transitions, we evaluated the relative stability during phase transition between two different phases (octahedral- $T$ and trigonal- $H$ ) of each composition, mimicked by the intralayer gliding of one of the chalcogen atom X planes relative to the metal atom $\mathrm{M}$ plane. We also explored the external strain effects on the phase transition by considering changes in activation energy barriers and reaction energies. We found that the external tensile strain may induce the phase transition in $\mathrm{MX}_{2}$ with $\mathrm{M}$ in the group 6. 


\section{Computational details}

To systematically investigate the structural stabilities and the electronic properties of various single-layered TMDCs, and the strain effect on their phase transitions, we used $a b$ initio density functional theory (DFT) [20] as implemented in the Vienna ab initio simulation packages (VASP). [21, 22] For the exchangecorrelation functional, we used the generalized gradient approximation (GGA) with the Perdew-Burke-Ernzerhof parameterization [23] with spin polarization. The local density approximation (LDA) was also used for comparison. Valence electrons were described under the projector augmented wave potentials, [24] and the electronic wave functions were expanded by a plane wave basis set with a cutoff energy of $400 \mathrm{eV}$. The corresponding Brillouin zone was sampled using a $\Gamma$-centered $50 \times 50 \times 1 \mathrm{k}$-grid mesh for the primitive unit cell. The charge density was determined self-consistently with a precision of $\leq 10^{-5} \mathrm{eV} /$ cell in total energy. A vacuum region of $\sim 22 \AA$ was included in a unit cell along a direction perpendicular to the $\mathrm{MX}_{2}$ plane to avoid the slab-slab interaction with the neighboring cells. All geometrical relaxations were continued until the Helmann-Feynman force acting on every atom had become lower than $0.01 \mathrm{eV} / \AA$. For heavy elements, such as Hf, Ta, and $\mathrm{W}$, we took into account the spin-orbit coupling (SOC) to describe their accurate electronic properties.

\section{Results and discussion}

It has been known that the TMDCs have a characteristic layered structure and can form different structural types due to the complex registry of atomic stacking sequence of metal and chalcogen atoms. [25] To perform a comparative study, we considered 18 different single-layered TMDCs. Each TMDC or MX $\mathrm{M}_{2}$ layer is composed of a plane of a transition metal element $\mathrm{M}(\mathrm{M}=\mathrm{Ti}, \mathrm{V}, \mathrm{Cr}, \mathrm{Zr}, \mathrm{Nb}, \mathrm{Mo}$, $\mathrm{Hf}, \mathrm{Ta}$, or $\mathrm{W})$ sandwiched by two planes of chalcogen element $\mathrm{X}(\mathrm{X}=\mathrm{S}$ or $\mathrm{Se})$. Depending on how to stack two chalcogen planes relative to each other, there are two representative possible configurations, trigonal-prismatic $(H)$ and octahedral (T) phases, which are known to be mostly observed in $\mathrm{MX}_{2}$ materials, [25] as shown in Fig. 1. The $H$ and $T$ phases are in resemblance to "ABA" and "ABC" stacking sequences in a stacked triangular lattice. Figure 1 clearly shows a difference between two phases. In the basal plane view of Fig. 1, both phases form a honeycomb lattice, which contains one $\mathrm{M}$ and two $\mathrm{X}$ atoms in the Wigner-Seitz cell.

For clear description of 18 different TMDC materials and their systematic analysis, we denoted each TMDC by a notation of $\mathrm{M}_{G}^{P} \mathrm{X}_{2}$, where the superscript 
$P$ and the subscript $G$ denote the period and the group to which the transition metal element $\mathrm{M}$ belongs in the periodic table, and $\mathrm{X}$ is either sulfur $(\mathrm{S})$ or selenium (Se) categorizing into sulfide or selenide. We further grouped the respective chalcogenides in terms of groups $G$ of $\mathrm{M}$ : $\mathrm{M}_{4}^{P}=\mathrm{Ti}, \mathrm{Zr}$, Hf for $P=4,5,6$, respectively, and similarly $\mathrm{M}_{5}^{P}=\mathrm{V}, \mathrm{Nb}$, Ta, and $\mathrm{M}_{6}^{P}=\mathrm{Cr}, \mathrm{Mo}, \mathrm{W}$. Figure 2 shows the calculated cohesive energies of the $H$ and $T$ phases of $\mathrm{M}_{G}^{P} \mathrm{X}_{2}$ as a function of lattice constants: (a), (b), and (c) are for sulfides, while (d), (e), and (f) are for selenides, of $\mathrm{M}_{4}^{P}, \mathrm{M}_{5}^{P}$, and $\mathrm{M}_{6}^{P}$, respectively, for three different $P$ values. Each energy point in Fig. 2 was obtained by finding the equilibrium structure for the corresponding lattice constant.

We found an overall common trend from Fig. 2 that TMDCs with a heavier TM element appear to be more stable than those with a lighter one within the same group $G$. It was, moreover, found that the group $G$, to which the transition metal atom $\mathrm{M}$ belongs, determines the equilibrium phases of $\mathrm{M}_{G}^{P} \mathrm{X}_{2}$ materials. As clearly shown in Fig. 2 (a) and (d), $\mathrm{M}_{4}^{P} \mathrm{X}_{2}$ materials prefer the octahedral $T$ phase (dotted line) to the $H$ counterpart (solid line). On the other hand, the materials with a metal atom $\mathrm{M}$ in the group $6(\mathrm{Cr}, \mathrm{Mo}$, and $\mathrm{W})$ favor the trigonal $H$ phase [7] over the $T$ counterpart, as shown in Fig. 2 (c) and (f). Intriguingly, the TMDCs with the group 5 metal atom $(\mathrm{V}, \mathrm{Nb}$, and $\mathrm{Ta})$ may coexist in both phases [7] as displayed in Fig. 2 (b) and (e). This result is consistent with a previous work finding coexistence of $\mathrm{VS}_{2}$ in both phases. [6] These results indicate that the stable phase of each TMDC is mainly determined by the group to which the transition metal belongs in the periodic table, as verified by the electronic occupation in its $d$ orbitals. [12] It is worth noting that the structural stability can be determined by free energy rather than cohesive energy. Nevertheless, to compare relative stability between two phases, one can use their cohesive energies if they have their corresponding single configurations. Since both $T$ and $H$ phases of any TMDCs are determined by their corresponding single equilibrium configurations, their cohesive energies may be good indicators to determine the relative stability.

Another interesting common trend was observed in the structural stiffness. As shown in Fig. 2, the energy curves for all the $T$ phases are flatter than their $H$ counterparts entailing that the former phases are softer than the latter. Such stiffness behavior can be observed more profoundly in tensile strain region, where $a$ is larger than the equilibrium lattice constant $a_{\text {eq }}$. Because of this stiffness trend, external stress applied on $\mathrm{M}_{4}^{P} \mathrm{X}_{2}$ (M in group 4) may not allow by itself their phase transition from their equilibrium $T$ phase to their $H$ counterpart. For $\mathrm{M}_{5}^{P} \mathrm{X}_{2}(\mathrm{M}$ in group 5), which can be in either phase near equilibrium, we expect the compounds in the $H$ phase may be converted into the $T$ phase by external tensile stress, but not 
the other way around. For $\mathrm{M}_{6}^{P} \mathrm{X}_{2}$ ( $\mathrm{M}$ in group 6), the energy curves representing two phases cross each other not only at a certain tensile strain value, but also at a certain compressive strain value, implying that external tensile (or compressive) stress may induce nearly spontaneous phase transition from their equilibrium $H$ phase to their $T$ counterparts, as we further discuss later. Figure 2 also shows that the equilibrium lattice constant $a_{\mathrm{eq}}$ of each $T$ phase tends to be almost the same as or a little larger than that of its $H$ counterpart, with the exception of $\mathrm{CrX}_{2}$, whose $T$-phase $a_{\text {eq }}$ is much larger than that of its $H$ phase. 
(a) $\mathrm{H}-\mathrm{MX}_{2}$ (Trigonal)
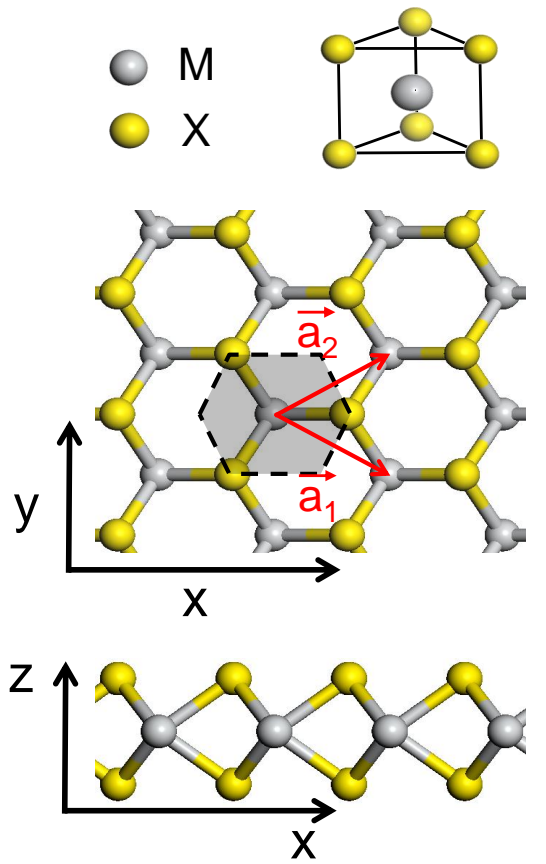

(b) $\mathrm{T}$-MX $\mathrm{M}_{2}$ (Octahedral)
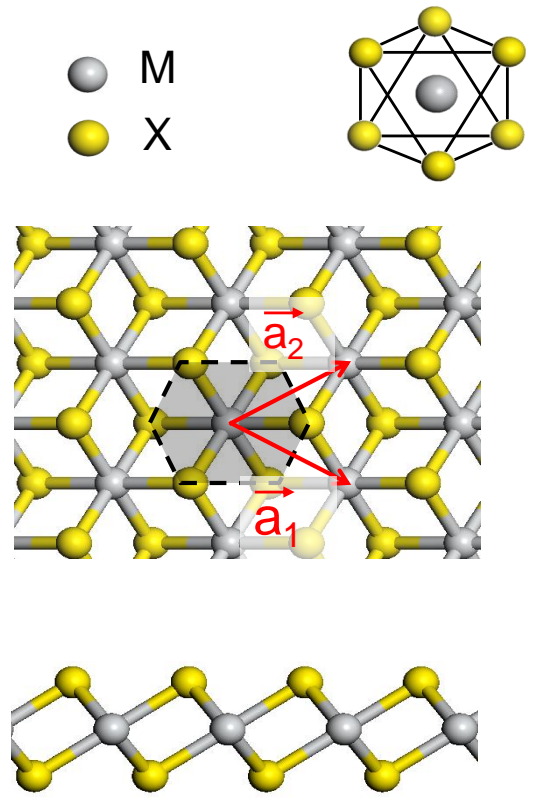

Figure 1: (Color online) Two different structural configurations of $\mathrm{MX}_{2}$ : (a) trigonal prismatic $(H$ phase) and (b) octahedral ( $T$ phase) configurations in basal plane and cross-section views together with their corresponding symmetries. Both $H$ and $T$ configurations form a hexagonal lattice composed of three atom basis of one transition metal atom $\mathrm{M}$ in the middle and two chalcogen atoms $\mathrm{X}$ and $\mathrm{X}^{\prime}$ on the sides with "ABA" and "ABC" atomic stacking sequences $\left(\mathrm{X}-\mathrm{M}-\mathrm{X}^{\prime}\right)$ in the WignerSeitz cell denoted by a gray- shaded region enclosed by a dashed hexagon. Two dimensional lattice vectors $\mathbf{a}_{1}$ and $\mathbf{a}_{2}$ are indicated by red arrows. Gray and yellow spheres depict transition metal (M) atoms and chalcogen $(\mathrm{X})$ atoms, respectively. 

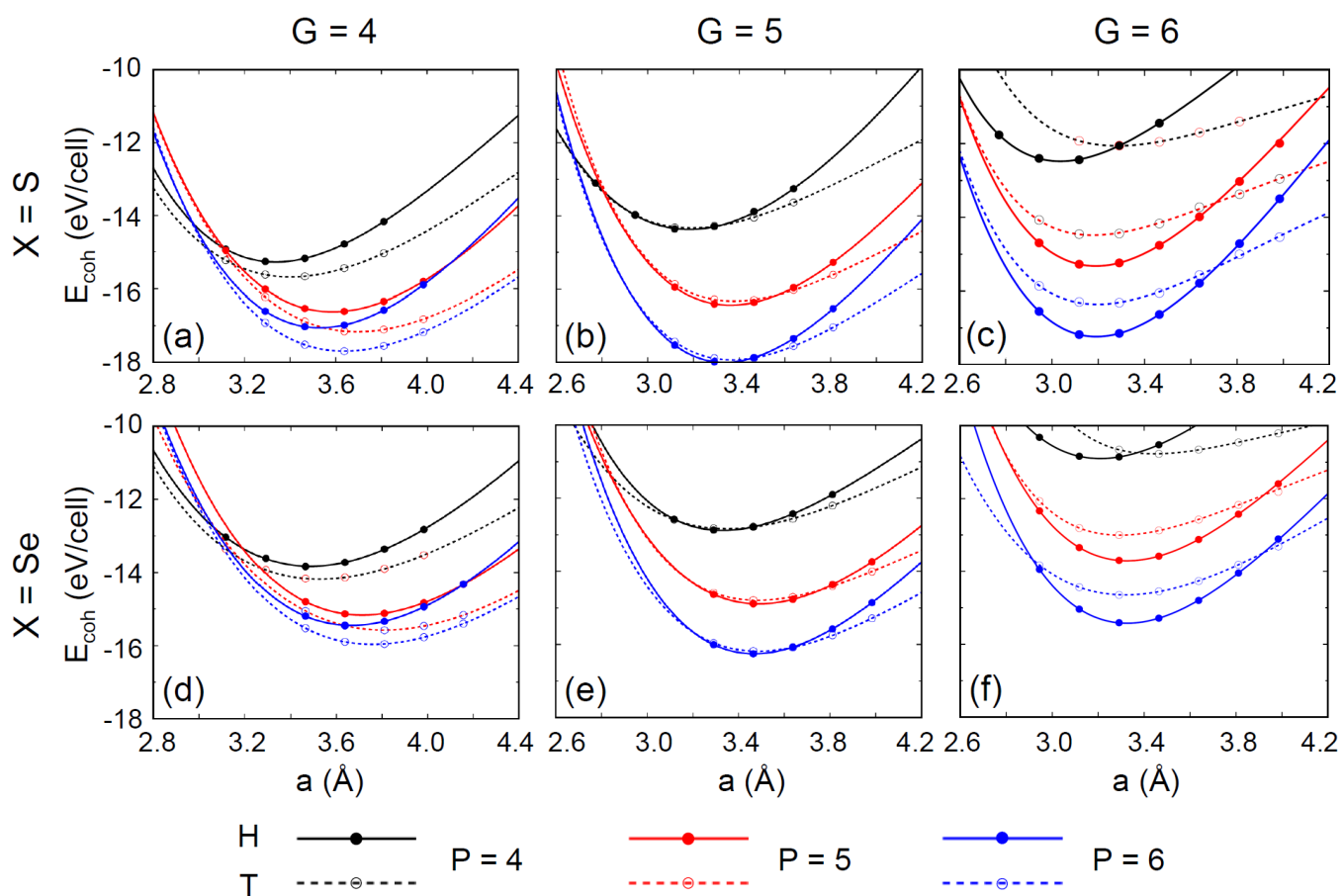

Figure 2: (Color online) Cohesive energy $E_{\text {coh }}$ of $\mathrm{M}_{G}^{P} \mathrm{X}_{2}$ as a function of lattice constants. Here, the superscript $P$ and the subscript $G$ denote respectively the period and the group to which M belongs in the periodic table: (a) $\mathrm{M}_{4}^{P} \mathrm{~S}_{2}$ (b) $\mathrm{M}_{5}^{P} \mathrm{~S}_{2}$, (c) $\mathrm{M}_{6}^{P} \mathrm{~S}_{2}$, (d) $\mathrm{M}_{4}^{P} \mathrm{Se}_{2}$, (e) $\mathrm{M}_{5}^{P} \mathrm{Se}_{2}$, and (f) $\mathrm{M}_{6}^{P} \mathrm{Se}_{2}$. In each figure, black, red, and blue lines represent $P=4,5$, and 6 , corresponding to $\mathrm{M}_{4}^{P}=(\mathrm{Ti}, \mathrm{Zr}$, Hf), $\mathrm{M}_{5}^{P}=(\mathrm{V}, \mathrm{Nb}, \mathrm{Ta})$, and $\mathrm{M}_{6}^{P}=(\mathrm{Cr}, \mathrm{Mo}, \mathrm{W})$, respectively; and solid and dotted lines delineate $E_{\text {coh }}$ of the $H$ and $T$ phases for a given $\mathrm{MX}_{2}$, as indicated by legend in the bottom. 

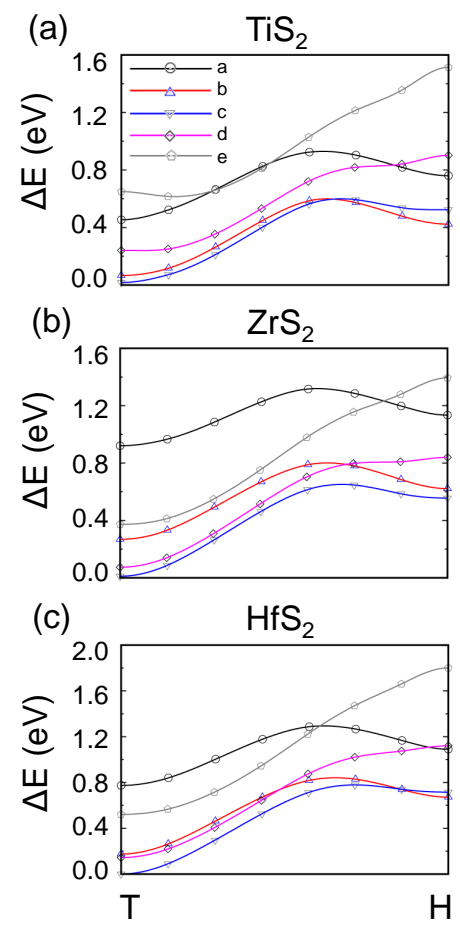

(d)

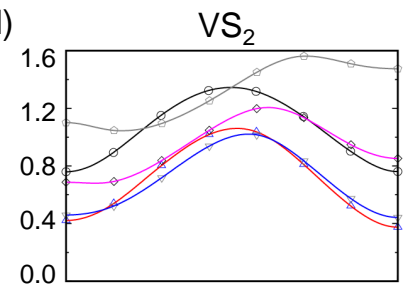

(e)

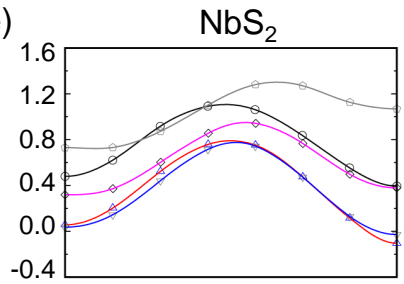

(f)

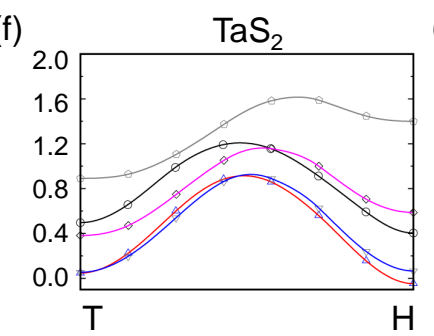

(g)

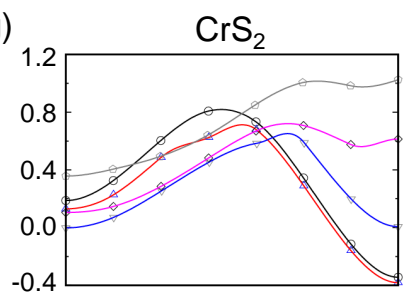

(h)

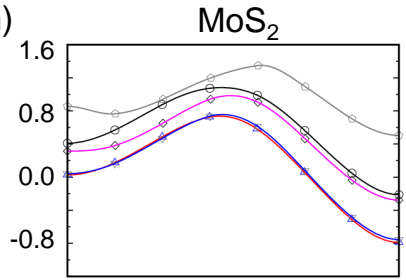

(i)

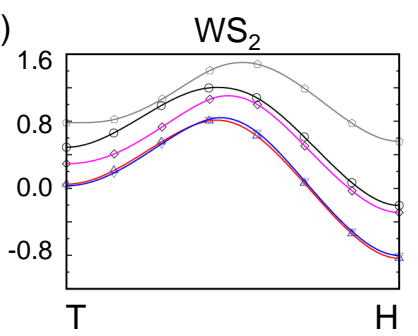

Figure 3: (Color online) Relative change in energy during the transition between the $T$ and the $H$ phases of all the sulfides: (a) $\mathrm{TiS}_{2}$ (b) $\mathrm{ZrS}_{2}$, (c) $\mathrm{HfS}_{2}$, (d) $\mathrm{VS}_{2}$, (e) $\mathrm{NbS}_{2}$, (f) $\mathrm{TaS}_{2}$, (g) $\mathrm{CrS}_{2}$, (h) $\mathrm{MoS}_{2}$ and (i) $\mathrm{WS}_{2}$ with various lattice constants indicated by ${ }^{a}$ through ${ }^{e}$, which correspond to in-plane strain values with the same indices listed in Tables 1 through 3 for sulfides. The relative energy values in each graph are referenced to the energy of the equilibrium $T$ phase of given $\mathrm{MS}_{2}$. The columns and rows of the graphs are arranged according to the groups and periods of the transition metal elements. 

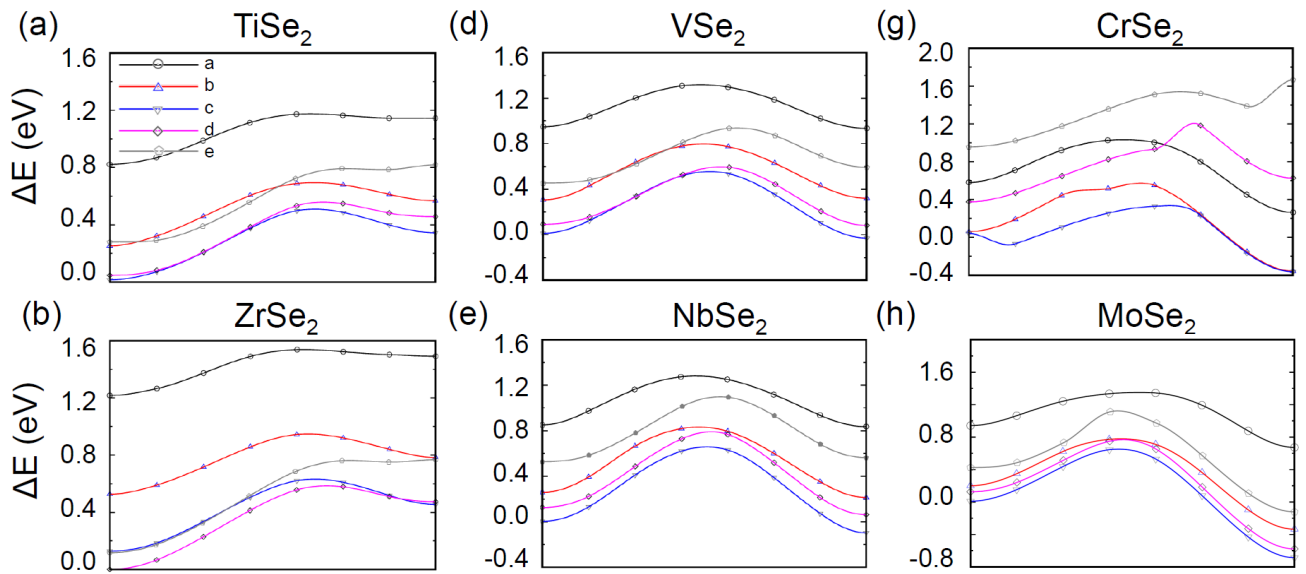

(e)

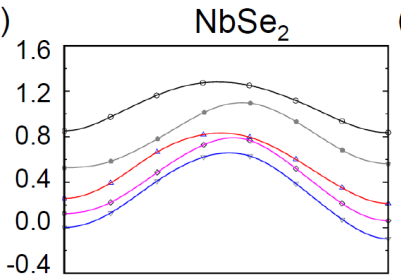

(h)
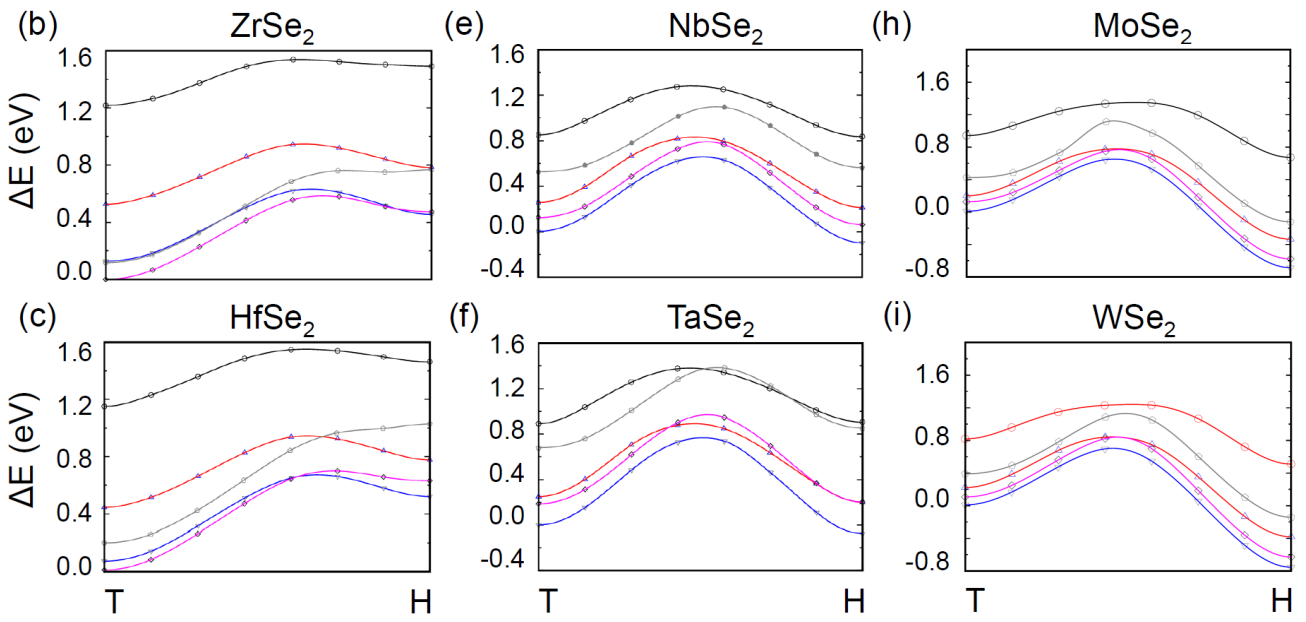

Figure 4: (Color online) Relative change in energy during the transition between the $T$ and the $H$ phases of all the selenides: (a) $\mathrm{TiSe}_{2}$ (b) $\mathrm{ZrSe}_{2}$, (c) $\mathrm{HfSe}_{2}$, (d) $\mathrm{VSe}_{2}$, (e) $\mathrm{NbSe}_{2}$, (f) $\mathrm{TaSe}_{2}$, (g) $\mathrm{CrSe}_{2}$, (h) $\mathrm{MoSe}_{2}$ and (i) $\mathrm{WSe}_{2}$ with various lattice constants indicated by ${ }^{a)}$ through ${ }^{e}$, which correspond to in-plane strain values with the same indices listed in Tables 1 through 3 for selenides. The relative energy values in each graph are referenced to the energy of the equilbrium $T$ phase of given $\mathrm{MSe}_{2}$. The columns and rows of the graphs are arranged according to the groups and periods of the transition metal elements. 
Table 1: For every $\mathrm{M}_{4}^{P} \mathrm{X}_{2}(\mathrm{X}=\mathrm{S}, \mathrm{Se})$ with several different lattice constants $(a)$, the corresponding in-plane strain values $(\epsilon)$, activation barriers $\left(\Delta E_{\mathrm{b}}\right)$ and reaction energies $\left(\Delta E_{\mathrm{r}}\right)$ are summarized. The same labels ${ }^{a)}$ through $^{e}$ ) were used as used in Figs. 3 and 4 . All the energy values are referenced to the energy of their respective $T$ phases. "-" marked in $\Delta E_{\mathrm{b}}$ indicates that the energy increases monotonically during the corresponding phase transition without the energy maximum.

\begin{tabular}{|c|c|c|c|c|c|c|c|c|c|c|c|c|c|}
\hline \multicolumn{2}{|c|}{$\mathrm{M}$} & \multicolumn{4}{|c|}{$\mathrm{Ti}$} & \multicolumn{4}{|c|}{$\mathrm{Zr}$} & \multicolumn{4}{|c|}{ Hf } \\
\hline $\mathrm{X}$ & $a(\AA)$ & & $\epsilon(\%)$ & $\Delta E_{\mathrm{b}}(\mathrm{eV})$ & $\Delta E_{\mathrm{r}}(\mathrm{eV})$ & & $\epsilon(\%)$ & $\Delta E_{\mathrm{b}}(\mathrm{eV})$ & $\Delta E_{\mathrm{r}}(\mathrm{eV})$ & & $\epsilon(\%)$ & $\Delta E_{\mathrm{b}}(\mathrm{eV})$ & $\Delta E_{\mathrm{r}}(\mathrm{eV})$ \\
\hline \multirow{6}{*}{$\mathrm{S}$} & 3.12 & a) & -8.34 & 0.48 & 0.31 & & & & & & & & \\
\hline & 3.29 & b) & -3.24 & 0.53 & 0.36 & a) & 10.72 & 0.40 & 0.21 & a) & -9.52 & 0.52 & 0.32 \\
\hline & 3.46 & c) & 1.85 & 0.58 & 0.51 & b) & -0.60 & 0.53 & 0.35 & b) & -4.75 & 0.66 & 0.50 \\
\hline & 3.64 & d) & 6.94 & - & 0.66 & c) & -1.32 & 0.64 & 0.55 & c) & 0.01 & 0.78 & 0.72 \\
\hline & 3.81 & e) & 12.03 & - & 0.87 & d) & 3.38 & - & 0.77 & d) & 4.77 & - & 0.98 \\
\hline & 3.98 & & & & & e) & 8.08 & - & 1.02 & $e)$ & 9.53 & - & 1.28 \\
\hline \multirow{6}{*}{$\mathrm{Se}$} & 3.12 & a) & -11.58 & 0.35 & 0.32 & & & & & & & & \\
\hline & 3.29 & b) & -6.67 & 0.44 & 0.32 & a) & -13.37 & 0.32 & 0.28 & a) & -12.46 & 0.40 & 0.31 \\
\hline & 3.46 & c) & -1.76 & 0.49 & 0.33 & b) & -8.81 & 0.42 & 0.26 & b) & -7.85 & 0.50 & 0.33 \\
\hline & 3.64 & d) & 3.16 & 0.51 & 0.41 & c) & -4.24 & 0.50 & 0.33 & c) & -3.25 & 0.60 & 0.45 \\
\hline & 3.81 & $e)$ & 8.07 & 0.51 & 0.54 & d) & 0.31 & 0.59 & 0.47 & d) & 1.36 & 0.69 & 0.62 \\
\hline & 3.98 & & & & & $e)$ & 4.16 & 0.64 & 0.65 & $e)$ & 5.97 & - & 0.82 \\
\hline
\end{tabular}


Table 2: For every $\mathrm{M}_{5}^{P} \mathrm{X}_{2}(\mathrm{X}=\mathrm{S}, \mathrm{Se})$ with several different lattice constants $(a)$, the corresponding in-plane strain values $(\epsilon)$, activation barriers $\left(\Delta E_{\mathrm{b}}\right)$ and reaction energies $\left(\Delta E_{\mathrm{r}}\right)$ are summarized. The same labels $\left.{ }^{a}\right)$ through ${ }^{e)}$ were used as denoted in Figs. 3 and 4 . All the reaction energy values are referenced to the energy of their respective $H$ phases. Negative activation barrier values indicate that the corresponding $T$ phase is more stable than its reference $H$ phase.

\begin{tabular}{|c|c|c|c|c|c|c|c|c|c|c|c|c|c|}
\hline \multicolumn{2}{|c|}{$\mathrm{M}$} & \multicolumn{4}{|c|}{$\mathrm{V}$} & \multicolumn{4}{|c|}{$\mathrm{Nb}$} & \multicolumn{4}{|c|}{$\mathrm{Ta}$} \\
\hline$X$ & $a(\AA)$ & & $\epsilon(\%)$ & $\Delta E_{\mathrm{b}}(\mathrm{eV})$ & $\Delta E_{\mathrm{r}}(\mathrm{eV})$ & & $\epsilon(\%)$ & $\Delta E_{\mathrm{b}}(\mathrm{eV})$ & $\Delta E_{\mathrm{r}}(\mathrm{eV})$ & & $(\%)$ & $\Delta E_{\mathrm{b}}(\mathrm{eV})$ & $\Delta E_{\mathrm{r}}(\mathrm{eV})$ \\
\hline \multirow{6}{*}{$\mathrm{S}$} & 2.94 & a) & -7.41 & 0.58 & 0.00 & & & & & & & & \\
\hline & 3.12 & b) & -1.96 & 0.69 & 0.05 & a) & -7.23 & 0.71 & 0.09 & a) & -6.55 & 0.80 & 0.09 \\
\hline & 3.29 & c) & 3.48 & 0.58 & 0.02 & b) & -2.08 & 0.90 & 0.16 & b) & -1.36 & 0.96 & 0.09 \\
\hline & 3.46 & d) & 8.93 & 0.35 & -0.17 & c) & 3.07 & 0.80 & 0.06 & c) & 3.83 & 0.86 & -0.01 \\
\hline & 3.64 & e) & 14.38 & 0.09 & -0.37 & d) & 8.22 & 0.57 & -0.06 & d) & 9.02 & 0.57 & -0.21 \\
\hline & 3.81 & & & & & e) & 13.38 & 0.23 & -0.34 & e) & 14.21 & 0.21 & -0.51 \\
\hline \multirow{6}{*}{$\mathrm{Se}$} & 2.94 & a) & -11.57 & 0.38 & 0.01 & & & & & & & & \\
\hline & 3.12 & b) & -6.36 & 0.48 & -0.01 & a) & -10.23 & 0.44 & 0.01 & a) & -6.55 & 0.48 & -0.01 \\
\hline & 3.29 & c) & -1.16 & 0.58 & 0.04 & b) & -5.66 & 0.62 & 0.04 & b) & -1.36 & 0.69 & 0.05 \\
\hline & 3.46 & d) & 4.04 & 0.51 & 0.01 & c) & -0.69 & 0.76 & 0.10 & c) & 3.83 & 0.84 & 0.08 \\
\hline & 3.64 & e) & 9.24 & 0.34 & -0.14 & d) & 4.27 & 0.73 & 0.06 & d) & 9.02 & 0.76 & -0.02 \\
\hline & 3.81 & & & & & $e)$ & 9.24 & 0.58 & -0.04 & e) & 14.21 & 0.53 & -0.17 \\
\hline
\end{tabular}


Table 3: For every $\mathrm{M}_{6}^{P} \mathrm{X}_{2}(\mathrm{X}=\mathrm{S}$, Se) with several different lattice constants $(a)$, the corresponding in-plane strain values $(\epsilon)$, activation barriers $\left(\Delta E_{\mathrm{b}}\right)$ and reaction energies $\left(\Delta E_{\mathrm{r}}\right)$ are summarized. The same labels $\left.{ }^{a}\right)$ through ${ }^{e}$ were used as used in Figs. 3 and 4 . The symbols $\dagger$ and ${ }^{\ddagger}$ correspond to the same symbols used in Fig. 5. All the energy values are referenced to the energy of their respective $H$ phases.

Negative activation barrier values indicate that the corresponding $T$ phase is more stable than its reference $H$ phase.

\begin{tabular}{|c|c|c|c|c|c|c|c|c|c|c|c|c|c|}
\hline \multicolumn{2}{|c|}{$\mathrm{M}$} & \multicolumn{4}{|c|}{$\mathrm{Cr}$} & \multicolumn{4}{|c|}{ Mo } & \multicolumn{4}{|c|}{$\mathrm{W}$} \\
\hline $\mathrm{X}$ & $a(\AA)$ & & $(\%)$ & $\Delta E_{\mathrm{b}}(\mathrm{eV})$ & $\Delta E_{\mathrm{r}}(\mathrm{eV})$ & & $\epsilon(\%)$ & $\Delta E_{\mathrm{b}}(\mathrm{eV})$ & $\Delta E_{\mathrm{r}}(\mathrm{eV})$ & & $\epsilon(\%)$ & $\Delta E_{\mathrm{b}}(\mathrm{eV})$ & $\Delta E_{\mathrm{r}}(\mathrm{eV})$ \\
\hline \multirow{7}{*}{ S } & 2.94 & a) & -3.03 & 1.16 & 0.53 & a) & -7.68 & 1.29 & 0.62 & a) & -7.74 & 1.41 & 0.69 \\
\hline & 3.12 & b) & 2.61 & 0.60 & -0.51 & b) & -2.25 & 1.53 & 0.82 & b) & -2.31 & 1.65 & 0.89 \\
\hline & 3.29 & c) & 8.38 & 0.65 & 0.01 & c) & 3.18 & 1.51 & 0.80 & c) & 3.11 & 1.64 & 0.83 \\
\hline & 3.46 & d) & 14.09 & 0.62 & 0.51 & d) & 8.61 & 1.26 & 0.59 & d) & 8.54 & 1.39 & 0.58 \\
\hline & 3.64 & e) & 19.79 & 0.04 & -0.67 & e) & 14.04 & 0.85 & 0.35 & e) & 13.97 & 0.94 & 0.22 \\
\hline & 3.81 & & & & & $\dagger$ & 19.47 & 0.31 & -0.34 & $\dagger$ & 19.29 & 0.43 & -0.29 \\
\hline & 3.98 & & & & & $\ddagger$ & 24.80 & 0.01 & -0.97 & $\ddagger$ & 24.62 & 0.06 & -1.04 \\
\hline \multirow{7}{*}{$\mathrm{Se}$} & 2.94 & a) & -8.25 & 0.77 & 0.32 & a) & -11.48 & 0.68 & 0.27 & a) & -11.47 & 0.73 & 0.31 \\
\hline & 3.12 & b) & -2.85 & 0.93 & 0.42 & b) & -6.27 & 1.11 & 0.54 & b) & -6.26 & 1.22 & 0.60 \\
\hline & 3.29 & c) & 2.55 & 0.70 & 0.41 & c) & -1.06 & 1.34 & 0.70 & c) & -1.05 & 1.46 & 0.76 \\
\hline & 3.46 & d) & 7.94 & 0.58 & -0.25 & d) & 4.15 & 1.35 & 0.70 & d) & 4.16 & 1.47 & 0.74 \\
\hline & 3.64 & e) & 13.34 & 0.16 & -0.43 & e) & 9.35 & 1.24 & 0.55 & e) & 9.37 & 1.27 & 0.53 \\
\hline & 3.81 & & & & & $\dagger$ & 14.46 & 0.74 & 0.26 & $\dagger$ & 14.48 & 0.95 & 0.23 \\
\hline & 3.98 & & & & & $\ddagger$ & 19.56 & 0.29 & -0.32 & $\ddagger$ & 19.59 & 0.40 & -0.28 \\
\hline
\end{tabular}


To study the strain effect on the phase transitions between $T$ and $H$ phases of TMDCs, we simulated the phase transition process of TMDC materials by sliding one of two chalcogen planes, which sandwich the transition metal plane, while applying compressive and tensile strain or adjusting lattice constant $a$ keeping the hexagonal lattice. (See Fig. S1(a) in Supplementary Information.) For each transition, we evaluated the relative energy with respect to the corresponding $T$ phase equilibrium configuration as a function of the relative coordinates of the shifted chalcogen atom. Our results for all $\mathrm{M}_{G}^{P} \mathrm{X}_{2}$ materials with $\mathrm{X}=\mathrm{S}$ and Se are shown in Figs. 3 and 4 , respectively, where each graph represents several relative energy curves plotted for different biaxial strains obtained by adjusting the lattice constant.

From these results, we extracted the corresponding in-plane strain values $(\epsilon)$, activation barriers $\left(\Delta E_{\mathrm{b}}\right)$ and reaction energies $\left(\Delta E_{\mathrm{r}}\right)$ are summarized in Tables 1 through 3. For each given lattice constant or in-plane strain in each $\mathrm{M}_{G}^{P} \mathrm{X}_{2}, \Delta E_{\mathrm{b}}$ values were evaluated by the energy difference from the lowest energy phase, either $T$ or $H$ phase, to the highest energy state between two phases, while $\Delta E_{\mathrm{r}}$ were obtained by the energy differences between two phases.

As clearly seen in Figs. 3 and 4 , and from Tables 1 through $3, E_{\mathrm{b}}$ values depend sensitively on change in the lattice constant $a$ or on the corresponding in-layer strain $\epsilon$. According to our calculation, $\mathrm{M}_{4}^{P} \mathrm{X}_{2}(\mathrm{X}=\mathrm{S}, \mathrm{Se})$ materials (M in group 4) in the $T$ phase become more stable than those in the $H$ phase as shown in Fig. 3 $(\mathrm{a}-\mathrm{c})$ and Fig. $4(\mathrm{a}-\mathrm{c})$. Moreover, we found that the larger tensile strain, the higher activation energy barrier and reaction energy during the transition to the $H$ phase as listed in Table 1, because of difference in their structural stiffness as already mentioned above. This indicates that any tensile stress may keep any TMDCs with a metal atom belonging to the $G=4$ group in the $T$ phase, but they may experience the phase transition to the $H$ phase at large compressive strain values.

Different transition behaviors were observed for $\mathrm{M}_{G}^{P} \mathrm{X}_{2}(\mathrm{X}=\mathrm{S}, \mathrm{Se})$ with $G=5$ and 6. It was found that for each case of $G=5$, either one of two phases is almost as stable as the other near the equilibrium and up to moderate compressive strain values, resulting in small reaction energies and admissible activation energy barriers, which depend less sensitively on strain values, as shown in Figs. 3 (d-f) and 4 (d-f), and as summarized in Table 2, than for the other cases of $G=4$ and $G=6$ shown in Tables 1 and 3 . Our result is in an agreement with a previous study on $\mathrm{VS}_{2}$ showing that small strain induces a phase transition from its $H$ phase to its $T$ counterpart. [6]

The most intriguing transition behaviors under external stress were found in $\mathrm{M}_{6}^{P} \mathrm{X}_{2}$ (M in group 6). Each material in this category is in the $H$ phase near 


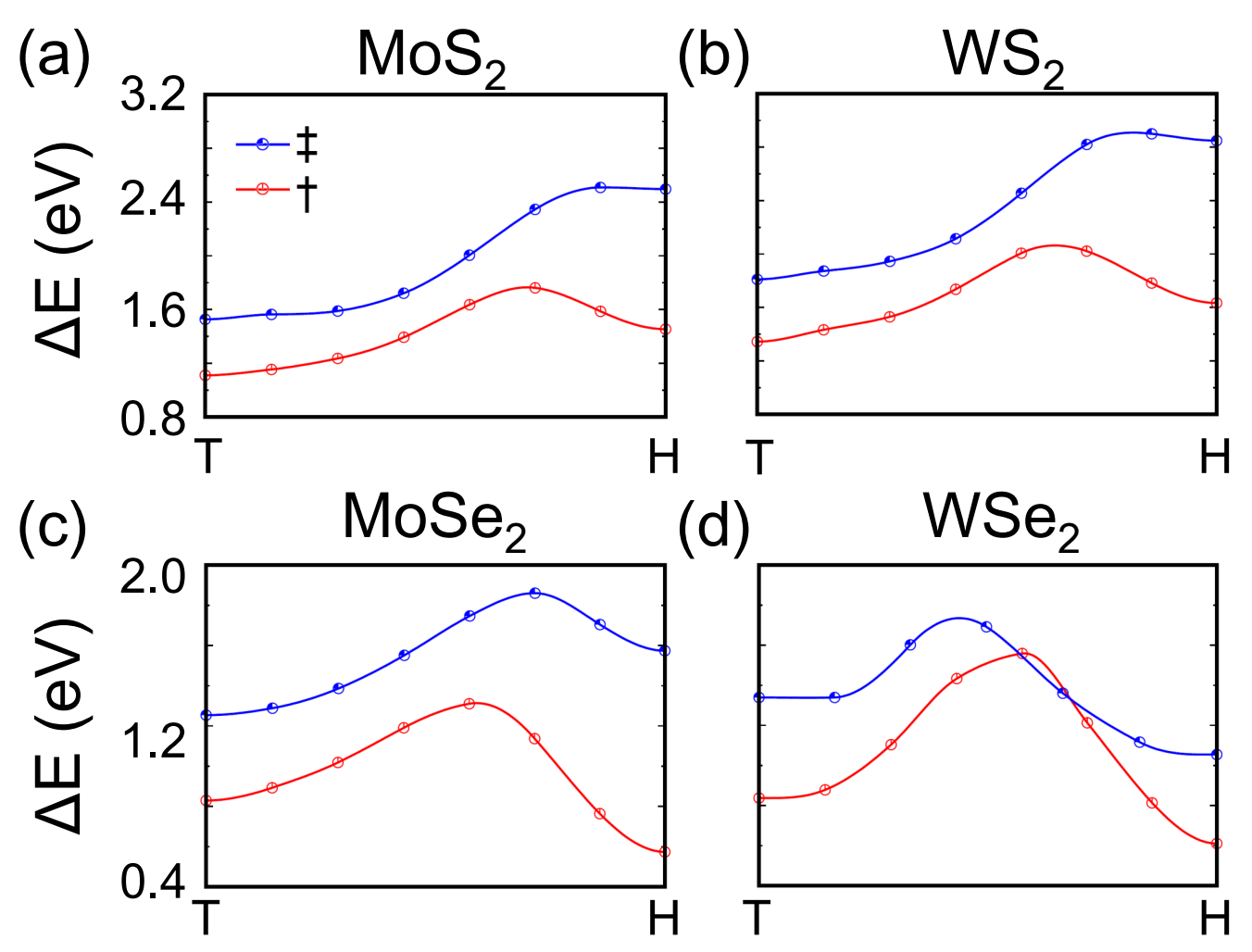

Figure 5: (Color online) Relative change in energy during the transition between the $T$ and the $H$ phases of (a) $\mathrm{MoS}_{2}$, (b) $\mathrm{WS}_{2}$ (c) $\mathrm{MoSe}_{2}$, and (d) $\mathrm{WSe}_{2}$ at two large lattice constants, $a=$ $3.81 \AA$ (red) and $3.98 \AA$ (blue) or large tensile strain values, indicated by ${ }^{\dagger}$ and ${ }^{\ddagger}$, which correspond to in-plane strain values with the same symbols listed in Table 3 .

equilibrium, but as external stress increases, its $T$ phase becomes more stable than its $H$ counterpart. Such phase crossover is clearly seen in Fig. 2(c) and (f), especially in the region of $a>a_{\mathrm{eq}}$ (tensile strain) for all cases and even in the region of $a<a_{\mathrm{eq}}$ (compressive strain) for a case of $\mathrm{WSe}_{2}$.

As shown in Figs. 3 ( $(\mathrm{g}-\mathrm{i}), 4(\mathrm{~g}-\mathrm{i})$, and 5 , and listed in Table 3 , the activation energy barrier becomes smaller with tensile strain applied although strain requires extra energy cost. Such energy barrier becomes even nearly zero at $20 \%$ or more in tensile strain, resulting in nearly spontaneous phase transition. Thanks to the bonding flexibility of S or Se atoms in TMDCs, the large tensile strain up to $20 \%$ may be achieved, [26] as similarly observed in Mo-S-I nanowires. [27, 28] Based on energy curves shown in Figs. 2 (c, f), and 5, we further estimated the energy cost for $\sim 20 \%$ strain to be about $0.33 \mathrm{eV}$ per each Mo-S bond, which corresponds 
to $33 \mathrm{GPa}$. We also noted, however, the structural stability under large tensile strain applied may not be guaranteed with a small unit cell as used in this study. Thus, we prepared much larger $6 \times 6$ supercell for $\mathrm{MoS}_{2}$ to realistically check the stability under large tensile strain. It was found that $\mathrm{MoS}_{2}$ maintains its symmetry and structure under tensile strain as large as near $20 \%$. This may provide a feasible microscopic explanation on experimental observations of pressure-induced insulator-metal transition of $\mathrm{MoS}_{2}$ implying the phase transition from its semiconducting $H$ phase to its metallic $T$ phase induced by strain. [17, 19]

To verify that our results on structural stability and strain dependence of phase transition are reliable, we further calculated with LDA. The relative energies calculated with LDA fall within only a few to ten percent ranges of those with GGA. In addition, we also considered SOC effect for heavy transition metal atoms, such as Hf, Ta, and W. For $\mathrm{WS}_{2}$, for instance, the equilibrium lattice constants of both $\mathrm{H}$ and $\mathrm{T}$ phases are essentially the same regardless of whether calculated with or without SOC. Moreover, the SOC effect does not change our finding that its $\mathrm{H}$ phase is more stable than its $\mathrm{T}$ counterpart, although the energy difference between two phases decreases significantly with SOC resulting in a narrow range of lattice constants where the $\mathrm{H}$ phase is more stable than the T phase. (See Fig. S1 (b) in Supplementary Information.) This means that phase transition could be achievable with smaller strain values than those we estimated above.

We further investigate the strain effects on the electronic properties of all $\mathrm{MX}_{2}$ materials in the $H$-and the $T$-phases. Earlier studies showed that the $H$ phase of $\mathrm{MoS}_{2}$ is semiconducting, [1] while its $T$ counterpart is metallic. [2] Figure 6 presents our results for the strain dependence of the electronic band gap of various $\mathrm{MX}_{2}$ materials. We divided our results into sulfides (a-c) and selenides (d-f), and collected them group by group to which the metal atoms belong, i.e. $\mathrm{M}$ in the group $4(G=4, \mathrm{Ti}, \mathrm{Zr}, \mathrm{Hf})$ in (a) and (d), the group $5(G=5, \mathrm{~V}, \mathrm{Nb}, \mathrm{Ta})$ in (b) and (e), and the group 6 ( $G=6, \mathrm{Cr}, \mathrm{Mo}, \mathrm{W})$ in (c) and (f).

Our calculation revealed that as shown in Fig. 6 (a, d) for the group 4, not only the $H$ phase, but also the equilibrium $T$ phase are semiconducting near their equilibria except for $\mathrm{TiS}_{2}$ and $\mathrm{TiSe}_{2}$, whose equilibrium $T$ phases are metallic. Intriguingly, even very small tensile strain triggers the metal-semiconductor transition in the $T$-phase $\mathrm{TiS}_{2}$, whereas it remains metallic under compressive strain. The $T$-phase $\mathrm{TiSe}_{2}$ becomes semiconducting at tensile strain above $\sim 5 \%$. In the other two metal cases ( $\mathrm{M}=\mathrm{Zr}$ and $\mathrm{Hf}$ ) in the group 4, their $T$ phases exhibit even larger energy band gap than their $H$ counterparts, regardless of whether $\mathrm{X}=\mathrm{S}$ or Se. Overall, the maximum band gap values of $\mathrm{M}_{4}^{P} \mathrm{X}_{2}$ fall within the ranges of $0.5-1.1 \mathrm{eV}$ for the $H$ phases near equilibrium or under small compressive strain, 

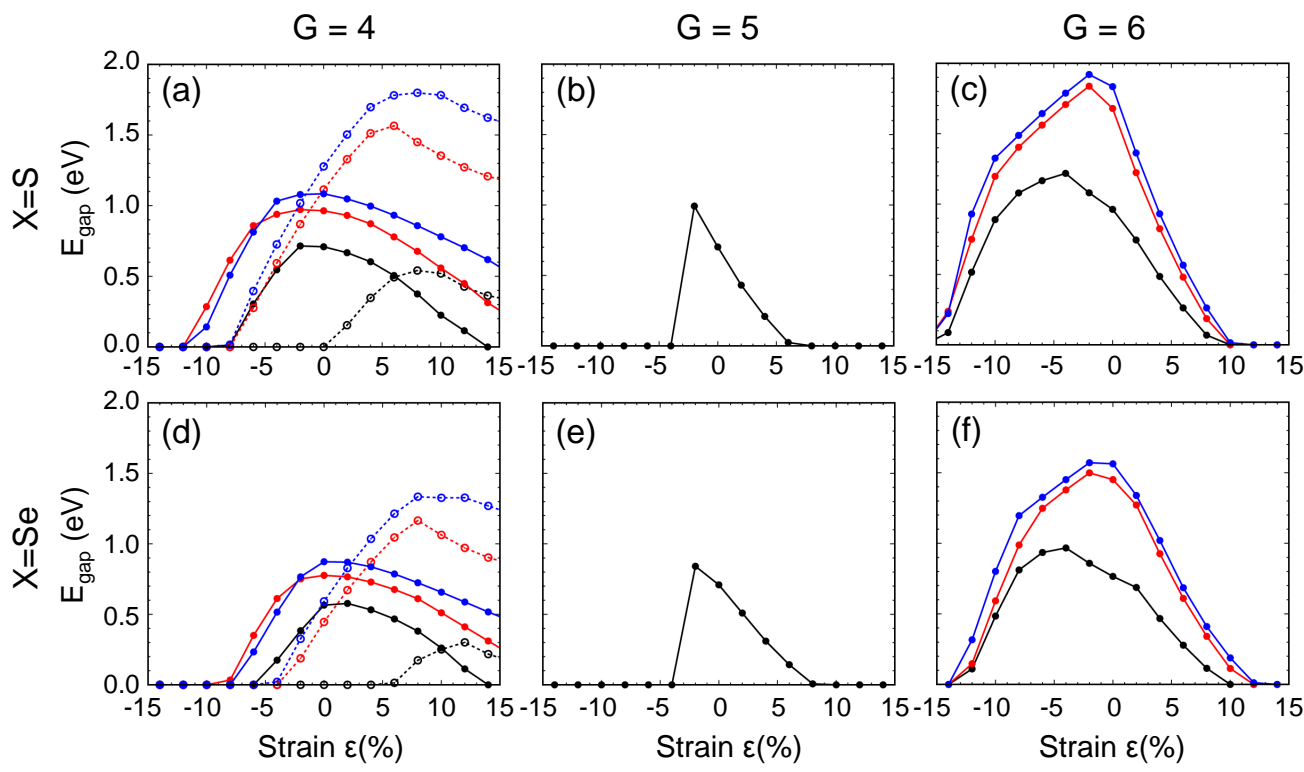

$(\mathrm{e})$
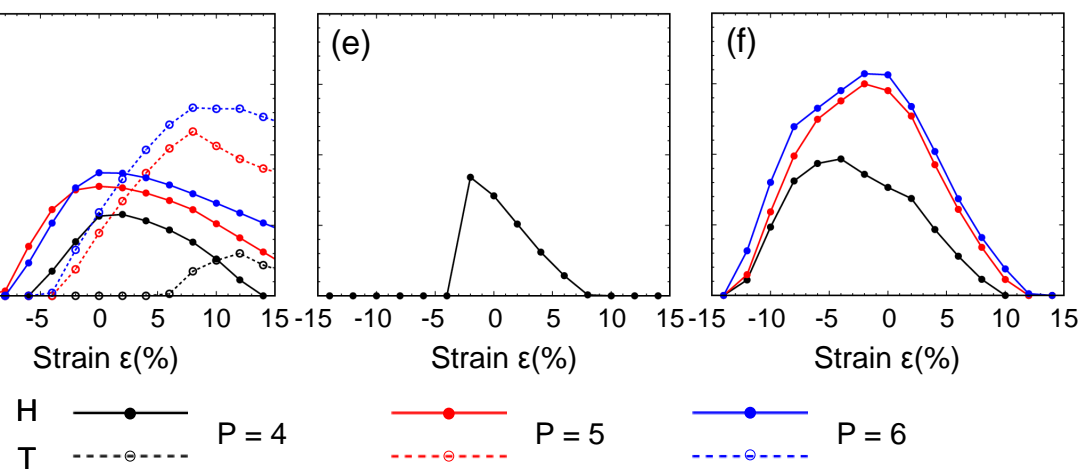

Figure 6: (Color online) Electronic band gap as function of strain in (a) $M_{4}^{P} S_{2}$ (b) $M_{5}^{P} S_{2}$, (c) $M_{6}^{P} S_{2}$, (d) $\mathrm{M}_{4}^{P} \mathrm{Se}_{2}$, (e) $\mathrm{M}_{5}^{P} \mathrm{Se}_{2}$, and (f) $\mathrm{M}_{6}^{P} \mathrm{Se}_{2}$. Black, red, and blue lines in each graph represents its band gap changes for $P=4,5$, and 6 , respectively, that is, $\mathrm{Ti}, \mathrm{Zr}$, and $\mathrm{Hf}$ for $G=4 ; \mathrm{V}, \mathrm{Nb}$, and Ta for $G=5$; and $\mathrm{Cr}$, Mo, and $\mathrm{W}$ for $G=6$. Solid lines indicate the band gap of the $H$ phases and dotted lines is $T$.

and of $1.2-1.8 \mathrm{eV}$ for the $T$ phases near $\sim 8 \%$ of tensile strain values. All the materials in this category eventually experience semiconducting-metal transitions under compressive strains.

For the group $5(G=5)$, all the $\mathrm{M}_{5}^{P} \mathrm{X}_{2}$ materials remain metallic under any strain values, whatever the chalcogen element $\mathrm{X}$ is, except for $\mathrm{VS}_{2}$ and $\mathrm{VSe}_{2}$ in the $H$ phase. Both exceptions are semiconducting within the strain range of $-5 \%<\epsilon \lesssim+8 \%$ with the maximum energy band gap of around $1 \mathrm{eV}$.

The group $6(G=6)$ cases exhibit interesting phase-dependent electronic characteristics: all the $T$-phase $\mathrm{M}_{6}^{P} \mathrm{X}_{2}$ materials remain metallic at any applied strain, while their $H$-phase counterparts experience their gap opening and closing as the applied strain increases from $-15 \%$ to $+15 \%$, as shown in Fig. 6 (c) and (f). They have the maximum band gaps near $\epsilon=0 \%$. To verify whether our calculated electronic structures are reliable, we performed more accurate calculation on $\mathrm{MoS}_{2}$ based on self-energy correction within the $G W$ approximation. 
We found that even in $G W$ calculations, its T phase remains metallic and its $H$ phase does semiconducting at their corresponding equilibria. For the latter case, its DFT-based band gap was underestimated by $\sim 1.4 \mathrm{eV}$ from its $G W$ value. (See Fig. S2 (a) in Supplementary Information.) Nonetheless, it turned out that band gap trends are similar between DFT and $G W$ calculations. Like the GGA result shown in Fig. 6 (c), the $G W$ band gap begins to close at $\sim 14 \%$ of tensile strain, which is larger than $\sim 10 \%$ in GGA. (See Fig. S2 (b) in Supplementary Information.) This indicates our observed insulator-metal transition may be robust and be induced by tensile strain.

\section{Conclusions}

In summary, we investigated the structural and electronic properties of $\mathrm{MX}_{2}$ ( $\mathrm{M}=\mathrm{Ti}, \mathrm{V}, \mathrm{Cr}, \mathrm{Zr}, \mathrm{Nb}, \mathrm{Mo}, \mathrm{Hf}, \mathrm{Ta}$, or $\mathrm{W} ; \mathrm{X}=\mathrm{S}$ or $\mathrm{Se}$ ) and its phase transition using density functional theory (DFT). We found that each group to which M belongs in the periodic table determines its characteristic equilibrium phase. The stable structural phases of group $4(\mathrm{Ti}, \mathrm{Zr}, \mathrm{Hf}$ ) are mainly octahedral, while those of group $6(\mathrm{Cr}, \mathrm{Mo}, \mathrm{W})$ are trigonal. Interestingly, those of group $5(\mathrm{~V}, \mathrm{Nb}, \mathrm{Ta})$ can be either one, since their two phase structures have almost the same total energy near their corresponding equilibrium lattice constants.

We also explored phase transitions between octahedral- $T$ and trigonal- $H$ phases for all $\mathrm{MX}_{2}$ compositions by evaluating the relative stability during their transition processes under various strain conditions. This exploration yielded the activation energy barriers between two phases as well as the reaction energies. Our results revealed that the compounds with $\mathrm{M}$ in the group 4 would experience phase transitions from their equilibrium $T$ phases to their $H$ counterpart under mainly compressive strains for $\mathrm{X}=\mathrm{S}$ and any strain conditions for $\mathrm{X}=\mathrm{Se}$. However, such transitioned phases would be converted back to their original equailibrium phases. Compounds with $\mathrm{M}$ in the group 5, which can exist in either the $T$ or the $H$ phase near their equilibrium or under compressive stress and would experience the phase transitions between two phases, tend to form the $H$ phases more easily under larger tensile stresses. Most interesting structural phase transitions were observed in compounds with $\mathrm{M}$ in the group 6 , which form the $H$ phases at their equilibria and hardly experience their phase transition to the $T$ phases due to large energy barrier values, but large tensile strain may make their transition to the $T$ phases relatively easily, and for some cases such transitions occur almost spontaneously with very small energy barriers. It was, moreover, found that external stress can be used to control the electronic properties, such as electronic energy 
gap, and eventually metal-semiconductor transition. Based on these results, we can surmise that the electron-phonon coupling may be an important property in some of $\mathrm{MX}_{2}$ materials, which will be considered as our future work.

\section{Acknowledgments}

We acknowledge financial support from the Korean government through National Research Foundation (NRF-2015R1A2A2A01006204, 2019R1A2C1005417) and the Ministry of Trade, Industry \& Energy (MOTIE) of Korea (Project No. 10045360). Some portion of our computational work was done using the resources of the KISTI Supercomputing Center (KSC-2016-C3-0034).

\section{References}

[1] L. F. Mattheiss, Phys. Rev. B 8 (1973).

[2] F. Wypych and R. Schollhorn, J. Chem. Soc. Chem. Commun. pp. 13861388 (1992).

[3] R. Bissessur, M. G. Kanatzidis, J. L. Schindler, and C. R. Kannewurf, J. Chem. Soc. Chem. Commun. pp. 1582-1585 (1993).

[4] G. Eda, T. Fujita, H. Yamaguchi, D. Voiry, M. Chen, and M. Chhowalla, ACS Nano 6, 7311 (2012).

[5] Y. Guo, D. Sun, B. Ouyang, A. Raja, J. Song, T. F. Heinz, and L. E. Brus, Nano Lett. 15, 5081 (2015).

[6] M. Kan, B. Wang, Y. H. Lee, and Q. Sun, Nano Res. 8, 1348 (2014).

[7] J. Wilson and A. Yoffe, Adv. Phys. 18, 193 (1969).

[8] A. D. Yoffe, Annu. Rev. Mater. Sci. 3, 147 (1973).

[9] A. Yoffe, Adv. Phys. 42, 173 (1993).

[10] B. Radisavljevic, a. Radenovic, J. Brivio, V. Giacometti, and a. Kis, Nat. nanotech. 6, 147 (2011).

[11] Q. H. Wang, K. Kalantar-Zadeh, A. Kis, J. N. Coleman, and M. S. Strano, Nat. nanotech. 7, 699 (2012). 
[12] M. Chhowalla, H. S. Shin, G. Eda, L.-J. Li, K. P. Loh, and H. Zhang, Nat. Chem. 5, 263 (2013).

[13] G. Eda, H. Yamaguchi, D. Voiry, T. Fujita, M. Chen, and M. Chhowalla, Nano Lett. 11, 5111 (2011).

[14] J. Heising and M. G. Kanatzidis, J. Am. Chem. Soc. 121, 638 (1999).

[15] F. Wypych, C. Solenthaler, R. Prins, and T. Weber, J. Solid State Chem. 144, 430 (1999).

[16] B. Ouyang, G. Lan, Y. Guo, Z. Mi, and J. Song, Appl. Phys. Lett. 107, 191903 (2015).

[17] Y.-C. Lin, D. O. Dumcenco, Y.-S. Huang, and K. Suenaga, Nat. nanotech. 9, 391 (2014).

[18] E. Scalise, M. Houssa, G. Pourtois, V. Afanasev, and A. Stesmans, Nano Res. 5, 43 (2011).

[19] A. P. Nayak, S. Bhattacharyya, J. Zhu, J. Liu, X. Wu, T. Pandey, C. Jin, A. K. Singh, D. Akinwande, and J.-F. Lin, Nat. Commun. 5, 3731 (2014).

[20] W. Kohn and L. J. Sham, Phys. Rev 385 (1965).

[21] G. Kresse and J. Furthmüller, Phys. Rev. B 54, 11169 (1996).

[22] G. Kresse and J. Hafner, Phys. Rev. B 47, 558 (1993).

[23] J. P. Perdew, K. Burke, and M. Ernzerhof, Phys. Rev. Lett. 77, 3865 (1996).

[24] G. Kresse and D. Joubert, Phys. Rev. B 59, 1758 (1999).

[25] M. Kertesz and R. Hoffmann, J. Am. Chem. Soc. 106, 3453 (1984).

[26] T. Li, Phys. Rev. B 85, 235407 (2012).

[27] S.-H. Kang, Y.-K. Kwon, and D. Tománek, J. Phys.: Condens. Matt. 22, 505301 (2010).

[28] S.-H. Kang, Y.-K. Kwon, and D. Tománek, Phys. Rev. B 82, 205427 (2010). 